\title{
Chemotherapy and irradiation in childhood Hodgkin's disease
}

\author{
B ROBINSON, J KINGSTON, R NOGUEIRA COSTA, J S MALPAS, A BARRETT, \\ AND T J McELWAIN
}

Section of Medicine, Institute of Cancer Research and Royal Marsden Hospital, Sutton and Department of Paediatric Oncology, St Bartholomew's Hospital, London

SUMMARY Eighty children aged less than 16 years with newly diagnosed Hodgkin's disease were treated between 1974 and 1982 . Complete remission occurred in $95 \%$, with actuarial five year overall survival of $94 \%$, and relapse free survival of $82 \%$ : median follow up was 4.8 years. Sixty one children were staged clinically while 19 had staging laparotomies before treatment. Most received combined modality treatment with Ch1VPP chemotherapy (chlorambucil, vinblastine, procarbazine, and prednisolone) followed by irradiation of initial bulk disease. Nodular sclerosis predominated in both sexes, accounting for $60 \%$ of the total. Girls with stage IV disease, nodal sclerosis histology, and bulky mediastinal masses had a relatively poor prognosis. Ten children have relapsed, and three prolonged (6 to 7 years) second remissions have been observed. Four died of disease, and one from infection. Clinical staging, avoiding splenectomy, reduced the risk of serious infections. Our current policy is to treat stage IA disease with local irradiation and all other stages with chemotherapy, adding irradiation for bulky mediastinal disease.

Children with Hodgkin's disease have an excellent prognosis with the prospect of long disease free survival in approximately $80 \% .{ }^{1-4}$ Staging techniques and treatment protocols that are used in adults, however, have been associated with significant morbidity and even mortality. Extended field irradiation which includes bony epiphyses compromises growth and frequently results in musculoskeletal deformity. ${ }^{235}$ Combination chemotherapy containing mustine causes distressing nausea and vomiting and leads to infertility in adult males ${ }^{6}$ and also in boys treated before or during puberty. ${ }^{78}$ Splenectomy in children with Hodgkin's disease is hazardous with the risk of fulminant septicaemia in up to $10 \% .{ }^{79}$ In addition there is an increased risk of a second primary malignancy in such children, especially those treated with a combination of extensive irradiation and chemotherapy. ${ }^{7}$

This report describes a new protocol, begun in September 1974, designed to avoid splenectomy and reduce irradiation fields and doses. Chemotherapy was given for both early and advanced disease, using Ch1VPP (chlorambucil, vinblastine, procarbazine and prednisolone), ${ }^{10}$ a combination causing much less nausea and vomiting than mustine-containing combinations. ${ }^{11}$

\section{Patients and methods}

From September 1974 to August 1982, 80 newly diagnosed children were staged and treated for Hodgkin's disease, and follow up continued to September 1983. Histology was classified by the Rye system. ${ }^{12}$ There were 56 boys and 24 girls (ratio $2 \cdot 3: 1$ ) whose median age was $10 \cdot 6$ years, (range $2 \cdot 8$ to 15.9 years).

Staging included physical examination, haematology profile, plasma liver function tests, and chest radiograph. Lymphography (with intravenous urogram) was undertaken provided there was no respiratory impairment. Lung tomography, computed tomography, ultrasound, and isotope scans were performed when indicated. Bone marrow o trephine was undertaken on 72 children, and lapar- N otomy was necessary for diagnosis in one child. In the first four years of the study, staging laparotomies were performed in 18 children before treatment-on completing therapy in six, at relapse in two, and for

This paper was prepared on behalf of the Children's Solid Tumour Group whose members are: A Barrett, H J G Bloom, J M Henk, J E Kingston, D N Lawson, T J McElwain, J S Malpas, S Meller, P N Plowman, and M R Sandland. 
other indications in one boy who did not have a splenectomy. Staging laparotomy included sampling of iliac, para-aortic, mesenteric, periportal, and splenic hilar lymph nodes; splenectomy; wedge biopsy of liver; and bone marrow trephine. Staging, by the Ann Arbor convention, ${ }^{13}$ was pathological in 19 children underging laparotomy before treatment, and clinical in the remainder. After splenectomy, prophylactic penicillin or erythromycin was given until at least 20 years of age.

\section{Treatment}

Treatment followed the Children's Solid Tumour Group protocol, which was modified during the first three years as laparotomy was phased out. Table 1 shows the final version. 'Bulk' disease was defined as a node mass with diameter greater than $5 \mathrm{~cm}$ or mediastinal mass greater than one third thoracic transverse diameter. Patients with clinical stage IA and IIA, with lymphocyte predominance, nodular sclerosis, or mixed cellularity, with not more than three affected sites and no bulk disease, were treated with Ch1VPP (three courses) followed by irradiation of affected sites (30 Gy over four weeks). Those with clinical stages I and II with lymphocyte depletion, bulk disease, or more than three affected sites, or with B symptoms, were treated with Ch1VPP (six courses) followed by irradiation of initial sites of disease (25 to $30 \mathrm{~Gy}$ in three to four weeks). Stage III patients were treated with Ch1VPP (six courses) followed by irradiation of sites of initial bulk disease only ( 25 to $30 \mathrm{~Gy}$ in three to four weeks). Stage IV patients were treated with $\mathrm{n}+$ five courses of Ch1VPP where $\mathrm{n}$ was the number

Table 1 Treatment protocol for childhood Hodgkin's disease

\begin{tabular}{|c|c|c|}
\hline \multirow{3}{*}{$\begin{array}{l}\text { Patient group } \\
\text { (Stage, histology, disease volume) } \\
\text { IA, not LD, small volume } \\
\text { IIA, not LD, small volume }<3 \text { sites }\end{array}$} & \multirow{2}{*}{\multicolumn{2}{|c|}{$\frac{\text { Treatment }}{\text { Chemotherapy Radiotherapy }}$}} \\
\hline & & \\
\hline & \multicolumn{2}{|c|}{$\begin{array}{ll}\text { ChIVPP* } & \text { Affected sites } \\
3 \text { courses } & 30 \mathrm{~Gy}\end{array}$} \\
\hline $\begin{array}{l}\text { IA, LD or large volume } \\
\text { IIA, LD or large volume or }>3 \text { sites } \\
\text { IB, all patients } \\
\text { IIB, all patients }\end{array}$ & $\begin{array}{l}\text { ChIVPP } \\
6 \text { courses }\end{array}$ & $\begin{array}{l}\text { Affected sites } \\
25-30 \mathrm{~Gy}\end{array}$ \\
\hline $\left.\begin{array}{l}\text { IIIA } \\
\text { IIIB }\end{array}\right\}$ all patients & $\begin{array}{l}\text { ChIVPP } \\
6 \text { courses }\end{array}$ & $\begin{array}{l}\text { Initial bulk sites } \\
25-30 \mathrm{GY}\end{array}$ \\
\hline $\left.\begin{array}{l}\text { IVA } \\
\text { IVB }\end{array}\right\}$ all patients & $\begin{array}{l}\text { ChIVPP } \\
6-10 \text { courses }\end{array}$ & $\begin{array}{l}\text { Mediastinal } \\
\text { bulk only } \\
30 \mathrm{GY}\end{array}$ \\
\hline
\end{tabular}

*Ch1VPP: chlorambucil $6 \mathrm{mg} / \mathrm{m}^{2}$ po days 1-14; vinblastine $6 \mathrm{mg} / \mathrm{m}^{2}$ IV days and 8 ; procarbazine $100 \mathrm{mg} / \mathrm{m}^{2}$ po days $1-14$; prednisolone $40 \mathrm{mg} / \mathrm{m}^{2}$ po days $1-14$.

$\mathrm{LD}=$ lymphocyte depletion. of courses to achieve complete remission. Those who did not remit after five courses were changed to alternative chemotherapy. Five of seven stage IV children with bulky mediastinal disease or pulmonary infiltration, or both, received irradiation to these sites after chemotherapy. All radiotherapy was administered using a 6 or $8 \mathrm{MeV}$ linear accelerator. Fifty five children were treated with chemotherapy followed by radiotherapy, 13 with chemotherapy alone, and 12 with radiotherapy alone. There were some deviations from the protocol in the first few years. Six children with pathological stage I and one with pathological stage II and small volume disease were treated with extended field irradiation, and two with pathological stage III disease with total nodal irradiation, to 35 and $40 \mathrm{~Gy}$ respectively. Four children (age less than 7 years) received additional courses of chemotherapy because radiotherapy fields would have included extensive growing bone.

Complete remission was defined as resolution of all abnormal symptoms, signs, and investigations attributable to Hodgkin's disease. Actuarial overall and relapse free survival rates were computed from diagnosis by standard Kaplan-Meier methods, and differences between curves by the log rank test.

Clinical presentation. Lymphadenopathy was a presenting symptom in 72 children $(90 \%)$ with enlargement of cervical or supraclavicular nodes in 51 $(63 \%)$. Constitutional symptoms of fever, sweats, or loss of more than $10 \%$ of body weight were noted in $17(21 \%)$. Thirteen $(16 \%)$ had splenomegaly, 7 $(8 \%)$ hepatomegaly, and $17(21 \%)$ were anaemic (haemoglobin less than $10 \mathrm{~g} / \mathrm{dl}$ ). None presented with superior vena caval obstruction. In eight diagnosis had been delayed by an earlier lymph node biopsy which had not shown Hodgkin's disease.

Histology and staging. Nodular sclerosis predominated in both girls $(75 \%)$ and boys $(54 \%)$ (Table 2 ). The male predominance $(2 \cdot 3: 1)$ and the incidence of nodular sclerosis in boys were both greater than in an earlier study from the Royal Marsden Hospital. ${ }^{11}$ Diesease stage distribution is shown in Table 2. Pathological staging upstaged six children and confirmed clinical staging in the remaining 13. Laparotomy showed that the spleen was affected in 10 , which in seven had not been detected clinically. One child with splenomegaly had a histologically normal spleen, but had clinical stage III disease with abdominal node involvement on lymphography (confirmed on biopsy). Although six children changed stage after laparotomy, different treatment was indicated in only three. One, upstaged from clinical stage IIA to pathological stage IIIA because 


\section{Barrett, and McElwain}

Table 2 Histology and disease stage of 80 children with Hodgkin's disease

\begin{tabular}{lccc}
\hline Histology & Boys & Girls & $\begin{array}{l}\text { Total } \\
\text { No (\%) }\end{array}$ \\
\hline Lymphocyte predominance & 11 & 2 & $13(16)$ \\
Nodular sclerosis & 30 & 18 & $48(60)$ \\
Mixed cellularity & 14 & 4 & $18(23)$ \\
Lymphocyte depletion & 1 & 0 & $1(1)$ \\
Total & 56 & 24 & $80(100)$ \\
\hline Disease stage & Clinical & Pathological & Total \\
& & & No (\%) \\
\hline IA & 10 & 6 & $16(20)$ \\
IIA & 24 & 2 & $26(33)$ \\
IIB & 5 & 0 & $5(6)$ \\
IIIA & 9 & 8 & $17(21)$ \\
IIIB & 3 & 0 & $3(4)$ \\
IVA & 3 & 1 & $9(5)$ \\
IVB & 7 & 2 & $80(100)$ \\
Total & & & \\
\hline
\end{tabular}

the spleen was affected, received six rather than three courses of chemotherapy, while two upstaged to pathological stage IV from clinical stages IIIB and IIA, were treated with chemotherapy alone without radiotherapy.

Routine marrow biopsy was not useful in staging. Only one of 72 had Hodgkin's disease infiltration and was already stage IV because the liver was affected.

\section{Results}

Response to treatment. Seventy six children (95\%), achieved complete remission after primary therapy. The complete remission rate was $100 \%$ for stages I and III, $94 \%$ for stage II, and $85 \%$ for stage IV. All 12 children treated with radiation alone achieved complete remission although one with nodular sclerosis histology showed slow resolution of mediastinal disease over nine months.

Forty nine of the 68 children who received CH1VPP were in complete remission at the end of chemotherapy. A further 17 were in remission except at sites of initial bulk disease, mediastinal in 15. After subsequent irradiation, a further 10 were in complete remission, but five showed slower resolution of mediastinal disease achieving complete remission one to two months after finishing radiotherapy in four and after nine months in the fifth. Complete remission was documented in 64 of 68 patients treated with CH1VPP, with or without irradiation.

Treatment failures occurred in three girls and one boy, all with nodular sclerosis histology and bulky mediastinal disease, two had clinical stage IIA disease and two clinical stage IVB. One died of $\stackrel{\rho}{?}$ disease, two are disease free after alternative chemotherapy, and one has active disease.

Toxicity of Ch1VPP. CH1VPP was well tolerated, with no alopecia and minimal nausea and vomiting. Antiemetics were not routinely given. Two children developed vinblastine-related ileus. Considerable modifications to protocol, that is delay of more than three weeks or dose reduction greater than $33 \%$, were made in 21 children-15 for haematological toxicity. Twelve had episodes of neutropenia, associated with anaemia or thrombocytopenia in seven and with infection in five (three serious). One 3 year old boy died in remission of clinical stage III disease, from Pneumocystis carinii pneumonia and pseudomonas septicaemia while neutropenic during $\vec{N}$ his final course of Ch1VPP. Procarbazine was $\overrightarrow{\vec{H}}$ stopped in four children for allergic skin reactions. At least $80 \%$ of protocol doses of vinblastine and chlorambucil were given to $95 \%$ of the children, while $75 \%$ received such doses of procarbazine.

Survival analysis. The actuarial overall survival rate at five years for all children is $94 \%$, with five year relapse free survival of $82 \%$ (Fig. 1). The five year relapse free survival rate is $100 \%$ for stage I, $85 \% \overrightarrow{0}$ for stage II, $77 \%$ for stage III, and $67 \%$ for stage I disease. Girls have a lower five year relapse freê survival rate $(69 \%)$ than boys $(88 \%)$, probabl related to the higher incidence of stage IV disease (38\% compared with $5 \%$ ). Lymphocyte predominant histology had the best prognosis with no relapses, but nodular sclerosis $(80 \%)$ had the same five year relapse free survival rate as mixed cellularity $(81 \%)$. The only child with lymphocyte depletion relapsed.

Relapses. Ten children (six boys, four girls) have relapsed, nine within three years of diagnosis, and one at seven years (Table 3). Seven relapsed in previously bulky sites of disease, either cervical nodes (three) or mediastinum (four), while three relapsed in previously unaffected sites. Both children with pathological stage III disease treated with irradiation relapsed, one in initially bulky cervical nodes: the other relapsed in extensive extradural sites and pelvic nodes seven years after $\tilde{N}$ radiotherapy - he died rapidly. Relapses were treated with Ch1VPP (four children) or alternative chemotherapy (four children, with irradiation in one), and irradiation alone in one child with recurrent initial bulky cervical nodes after primary treatment with Ch1VPP. Seven relapsers are alive and three dead. Five have been in a second complete recession since treatment of first relapse, 


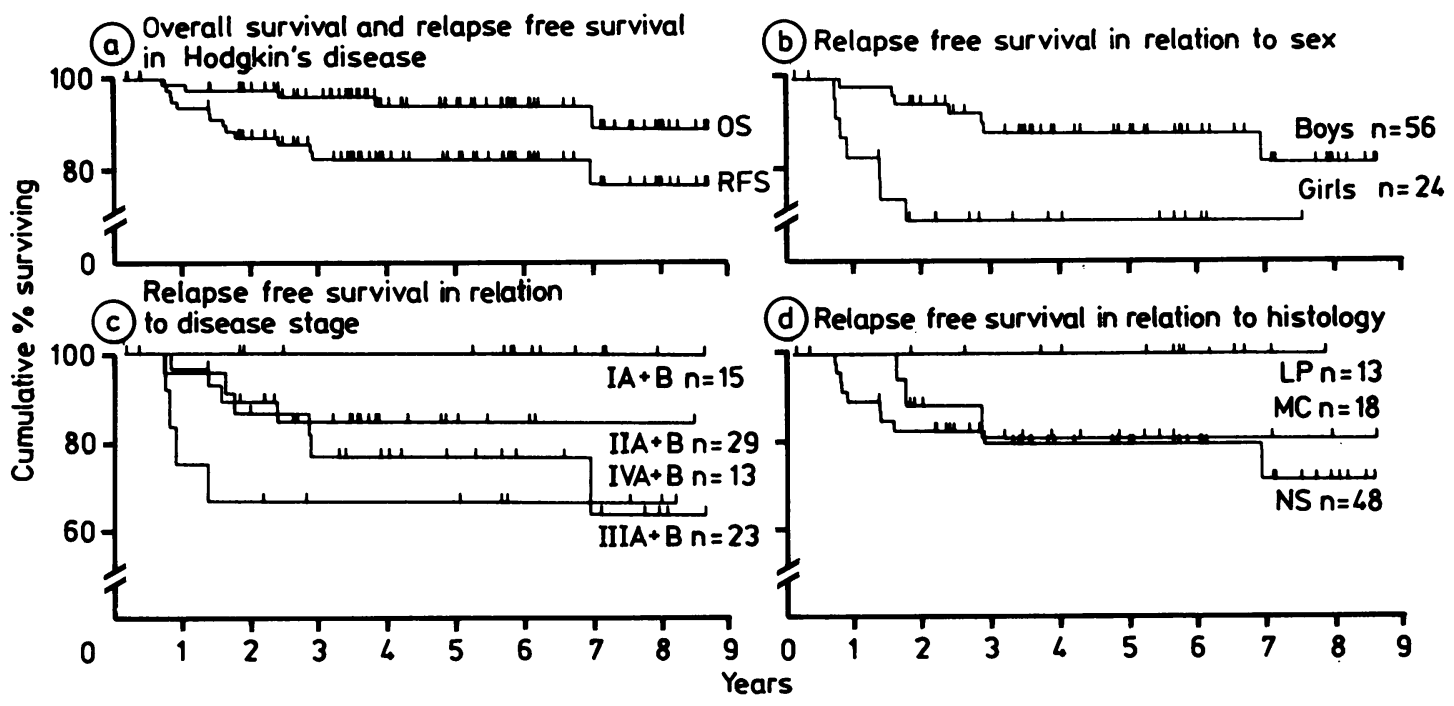

Fig. 1 Overall and relapse free survival and relapse free survival to sex, disease stage and histology in 80 children with Hodgkin's disease.

$\mathrm{LP}=$ Lymphocyte predominance $\mathrm{MC}=$ mixed cellularity $\mathrm{NS}=$ nodular sclerosis.

Table 3 Relapses after primary treatment in 10 patients

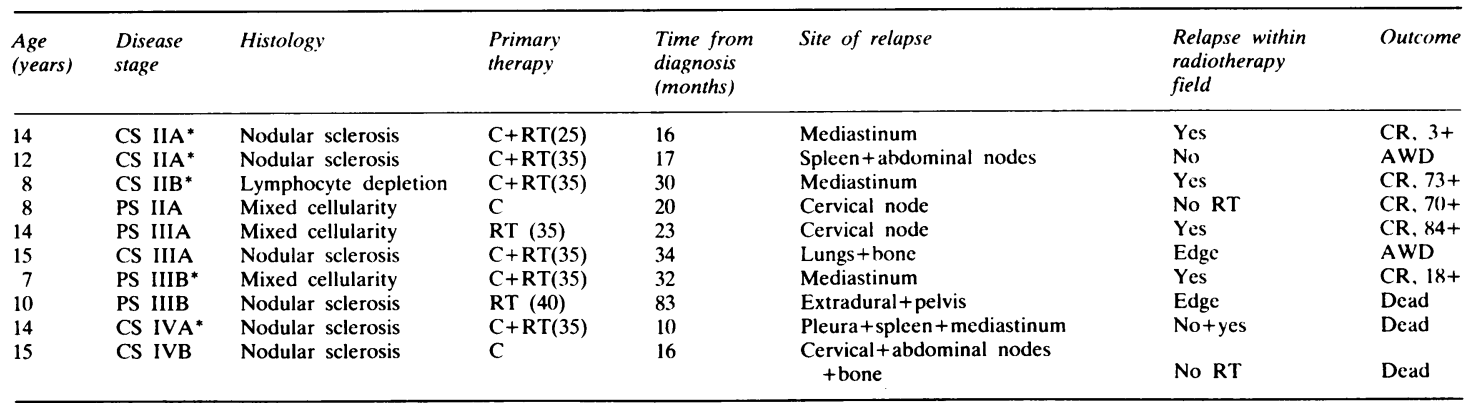

$\mathrm{CS}=$ clinical stage $\mathrm{PS}=$ pathological stage $\mathrm{C}=\mathrm{Ch} 1 \mathrm{VPP}$ chemotherapy, $\mathrm{RT}=$ radiotherapy $($ dose in $\mathrm{Gy}) ; \mathrm{AWD}=$ alive with disease $: \mathrm{CR}=$ complete remission since 1st relapse, duration in montlis.

*Mediastinal mass greater than one third thoracic transverse diameter.

three for six to seven years, while two have active disease. The salvage rate for relapse after combined modality therapy was three of six, and two of four for single modality therapy, or $50 \%$ overall.

Effect of mediastinal bulk. Bulky mediastinal disease (mediastinal mass greater than one third thoracic transverse diameter) was present in 28 children and had a lower five year relapse free survival rate $(70 \%$ compared with $90 \%$ ) than disease with an $\mathrm{M}: \mathrm{T}$ ratio of less than 1:3 (Fig. 2). This difference was just significant at the $10 \%$ level with $P=0 \cdot 088$. Twenty

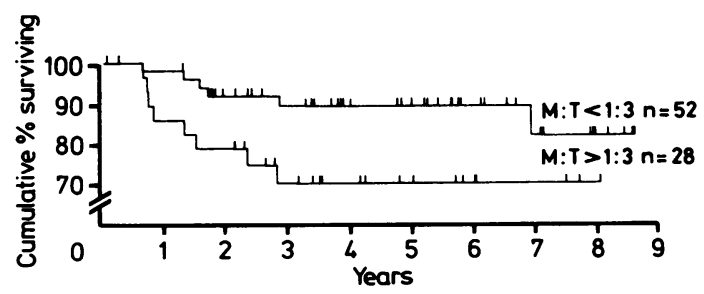

Fig. 2 Relapse free survival in children with bulky mediastinal disease (mediastinal mass greater than one third thoracic transverse diameter $(M: T>1: 3))$. 
Table 4 Serious infections in children with Hodgkin's disease

\begin{tabular}{|c|c|c|c|c|c|}
\hline \multicolumn{3}{|c|}{ Splenectomy $(n=27)$} & \multirow{2}{*}{$\begin{array}{l}\text { Months since } \\
\text { splenectomy }\end{array}$} & \multicolumn{2}{|c|}{ No splenectomy $(n=53)$} \\
\hline $\begin{array}{l}\text { Patient } \\
\text { No }\end{array}$ & Infections & & & $\begin{array}{l}\text { Patient } \\
\text { No }\end{array}$ & Infections \\
\hline \multirow[t]{3}{*}{1} & Wound infection & & ${ }^{*}+<1$ & 1 & Pneumocystis carinii \\
\hline & Streptococcus pneumoniae septicaemia & & 3 & & + pseudomonas septicaemiat \\
\hline & Pneumonia & & 5 & & Died \\
\hline 2 & Pneumonia & & $<1$ & 2 & $\beta$ haemolitic streptococcal endocarditis \\
\hline 3 & $\begin{array}{l}\text { Pseudomonas pneumonia and } \\
\text { septicaemiat }\end{array}$ & · & 4 & 3 & $\begin{array}{l}\text { Pneumonia } \\
\text { Pneumonia }\end{array}$ \\
\hline \multirow[t]{2}{*}{4} & Pneumonia $\dagger$ & & 7 & & \\
\hline & Pneumonia & & 9 & & \\
\hline 5 & Wound infection & & $<1$ & & \\
\hline
\end{tabular}

*Patient discontinued penicillin; tneutropenia

three children with mediastinal disease received Ch1VPP chemotherapy and mediastinal irradiation ( 25 to $30 \mathrm{~Gy}$ in 13 and 35 to $40 \mathrm{~Gy}$ in 10 ) while five (all stage IV) received Ch1VPP alone. Three failed to remit and five relapsed, a failure rate of $29 \%$, and only three of these eight are free of further recurrences. In six, the mediastinum was the site of disease progression and had been irradiated in five, with 35 to $40 \mathrm{~Gy}$ in four and $25 \mathrm{~Gy}$ in one. All 14 with stage II disease and mediastinal mass greater than one third thoracic transverse diameter received combined modality treatment, yet three relapsed $(21 \%)$-two in the mediastinum and one infradiaphragmatic-while another failed to remit with persisting mediastinal disease.

Complications of treatment. Life threatening infections with septicaemia occurred in five children, three after splenectomy (Table 4). The risk of bacterial infection was significantly greater after splenectomy, 0.33 , compared with 0.08 for no splenectomy, $\mathbf{P}<0 \cdot 01$. Even when three postoperative infections are excluded, the risk after splenectomy is $0 \cdot 22$, just significant at the $10 \%$ level. Herpes zoster and varicella infections occurred with similar frequency whether splenectomy was performed $(0 \cdot 33)$ or not $(0 \cdot 38)$. Nine patients developed herpes zoster during treatment and 14 after completing this, eight within the first year.

Most children are now postpubertal. Two girls have secondary amenorrhoea and one girl failed to enter puberty after TNI, and Ch1VPP for relapse. Two girls aged 13 and 15 years given 10 courses of Ch1VPP have had normal offspring. Three boys have azoospermia, two after nine and ten courses of Ch1VPP given at age 15 and 12 years respectively, and one after only three courses of Ch1VPP (and neck irradiation) given at age 13 years. Eight children have deformity of the thorax (that is, small clavicles, shortened thoracic spine, or pectus de- formity) or atrophy of soft tissue of the neck, or $\overrightarrow{\hat{N}}$ both, after irradiation at ages 11 to 14 years. All had received 35 to $40 \mathrm{~Gy}$ to the mediastinum or modified $\mathbb{N}$ mantle field. No second malignancies have de- o veloped.

\section{Discussion}

A complete remission rate of $95 \%$ was achieved in 80 children with Hodgkin's disease, all stages. The five year relapse free survival rate was $82 \%$, with $\$$ five year overall survival of $94 \%$ and a media follow up of 4.8 years. These results, similar to thoss of several recent reports, ${ }^{3-5} 1114$ were obtaine ${ }^{+}$ using a protocol designed to reduce management related morbidity. Alopecia was avoided and vomiting reduced by Ch1VPP chemotherapy. ${ }^{10}$ Irradiation doses were reduced to 25 to $30 \mathrm{~Gy}$ and fields limited to sites of initial bulk disease to minimise effects on bone growth. Chemotherapy for early disease enabled staging laparotomy with splenectomy to be abandoned since bulky sites were detected clinically and occult disease would be controlled by chemotherapy.

It is now clear that children with stage IA disease in high cervical or inguinal nodes do not often have clinically unsuspected abdominal disease and rarely relapse after irradiation alone, ${ }^{1515}$ with any such relapses readily salvaged by chemotherapy. Thus, clinical stage IA patients treated with Ch1VPP and irradiation in this study were certainly overtreated and stage II patients with only two affected sites and small volume disease (less than $5 \mathrm{~cm}$ diameter) probably also received unnecessary chemotherapy. When bulky stage I and II disease in children is treated with irradiation, however, there is a 20 to $30 \%$ relapse rate $^{2416}$ which approaches $50 \%$ in those with massive mediastinal disease (mediastinal mass greater than one third thoracic transverse diameter). ${ }^{14}{ }^{17}$ Although combined modality treatment 
of bulky mediastinal disease has a lower relapse rate, for example $21 \%$ for stage II disease in this study, relapses remain a problem and overall survival is not affected. ${ }^{14}$ Our study confirms the poor prognosis of bulky mediastinal disease,${ }^{14}$ with $70 \%$ for five year relapse free survival and a low salvage rate $(38 \%)$ despite chemotherapy or combined modality treatment. Stage III disease in children is inappropriately treated by irradiation alone $;^{2-4} 16$ both our patients relapsed compared with three of 18 treated with chemotherapy with or without radiotherapy. The group with stage IV disease included more children with nodular sclerosis histology (12 children or $92 \%)$, girls $(8,69 \%)$, and bulky mediastinal disease $(8,62 \%)$ than the whole group, and these features were associated with a poor prognosis.

Infections were few without splenectomy. After splenectomy serious sepsis occurred despite antibiotic prophylaxis, emphasising that although use of prophylactic antibiotics and pneumococcal vaccination (not done here) reduce the incidence of sepsis after splenectomy to less than $5 \%$, a significant risk remains because of non-compliance, failure to cover all pathogens, and variability of immune responses. ${ }^{18}{ }^{19}$ No second malignancies have developed in these 80 children, but these have occurred in earlier cohorts. ${ }^{11} 15$ From other studies, the risk seems less than in adults, and higher after combined modality treatment with extensive irradiation or treatment for relapse. ${ }^{1720}$ Continued follow up is essential to determine whether greater use of chemotherapy will increase the risk of acute leukaemia.

This experience forms the basis for a new national protocol sponsored by the United Kingdom Children's Cancer Study Group. This employs clinical staging without laparotomy. Children with clinical stage IA disease with cervical or inguinal nodes affected only (excluding lymphocyte depletion histology) are treated with local radiotherapy to a dose of $30 \mathrm{~Gy}$. Boys so treated will not be sterilised. Chemotherapy is now so effective that we expect it to reclaim any relapses.

All other patients are treated with Ch1VPP chemotherapy, with addition of low dose irradiation only for bulky mediastinal disease. New approaches are still needed for the small minority who fail with current treatment, and efforts must continue to develop even less toxic treatments, particularly those with no risk of carcinogenesis, the major potential long term problem in this approach to treatment.

We thank colleagues who referred patients, staff of paediatric departments at the Royal Marsden and St Bartholomew's Hospitals, and Mrs Pauline Holloway, Research Secretary for the
Children's Solid Tumour Group. BAR is supported by the Nuffield Foundation and TJM by the Cancer Research Campaign and the Medical Research Council.

\section{References}

' Jenkin D, Chan H, Freedman M, et al. Hodgkin's disease in children: treatment results with MOPP and low-dose extended field irradiation. Cancer Treat Rep 1982;66:949-59.

2 Lange B, Littman P. Treatment of Hodgkin's disease in children and adolescents. Cancer 1983;51:1371-7.

${ }^{3}$ Mauch PM, Weinstein H, Botnick L, Belli J, Cassady JR. An evaluation of long-term survival and treatment complications in children with Hodgkin's disease. Cancer 1983;51:925-32.

${ }^{4}$ Tan C. Jereb B, Chan KW, Lesser M, Mandora A, Exelby P. Hodgkin's disease in children. Results of management between 1970 and 1981. Cancer 1983;51:1720-5.

5 Jenkin D, Berry M. Hodgkin's disease in children. Semin Oncol 1980:7:202-11.

${ }^{6}$ Chapman RM, Sutcliffe SB, Malpas JS. Cytotoxic induced ovarian failure in women with Hodgkin's disease. JAMA 1979;242:1877-81.

${ }^{7}$ Donaldson SS, Kaplan HS. Complications of treatment of Hodgkin's disease in children. Cancer Treat Rep 1982;66: 977-89.

* Whitehead E, Shalet SM, Morris-Jones PH, Beardwell CG, Deaken DP. Gonadal function after combination chemotherapy for Hodgkin's disease in childhood. Arch Dis Child 1982;47:287-91.

${ }^{9}$ Chilcote RR, Baehner RL, Hammond D. Septicaemia and meningitis in children splenectomised for Hodgkin's disease. $N$ Engl J Med 1976;295:798-800.

10 McElwain TJ, Toy J, Smith IE, Peckham MJ, Austin DE. A combination of chlorambucil, vinblastine, procarbazine and prednisolone for treatment of Hodgkin's disease. Br J Cancer 1977:36:276-80.

1 Smith IE, Peckham MJ, McElwain TJ, Austin DE. Hodgkin's disease in children. Br J Cancer 1977;36:120-9.

12 Lukes RJ, Craven LF, Hall TC, Rappaport H, Ruben P. Report of the Nomenclature Committee. Cancer Res 1966;26:1311.

13 Carbone PP, Kaplan HS, Musshoff K, Smithers DW, Tubiana M. Report of the committee on Hodgkin's disease Staging Classification. Cancer Res 1971:31:1860-1.

14 Roskos RS, Evans RC, Gilchrist GS, Burgert EO, Ilstrup DM. Prognostic significance of mediastinal mass in childhood Hodgkin's disease. Cancer Treat Rep 1982;66:961-8.

15 Malpas JS. Lymphomas in children. Semin Hematol 1982;19:301-14.

16 Jenkin D, Freedman M, McClure P, Peters V, Saunders F, Sonley M. Hodgkin's disease in children: treatment with low dose radiation and MOPP without staging laparotomy: a preliminary report. Cancer 1979;44:80-6.

17 Green DM, Ghoorah J, Douglass HO, et al. Staging laparotomy with splenectomy in children and adolescents with Hodgkin's disease. Cancer Treat Rev 1983;10:23-38.

is Addiego JE, Ammann AJ, Schiffman G, Baehner R, Higgins G, Hammond D. Response to pneumococcal vaccine in patients with untreated Hodgkin's disease. Lancet 1980;ii:450-2.

${ }_{19}$ Donaldson SS, Vosti KL, Berberich FR, Cox RS, Kaplan HS, Schiffman G. Response to pneumococcal vaccine among children with Hodgkin's disease. Rev Infect Dis 1981;3 (Suppl):S133-43.

${ }^{20}$ Krikorian JG, Burke JS, Rosenberg SA, Kaplan HS. Occurrence of non-Hodgkin's lymphoma after treatment for Hodgkin's disease. $N$ Engl J Med 1979;300:452-8.

Correspondence to Professor T J McElwain, Institute of Cancer Research, Royal Marsden Hospital, Sutton, Surrey.

Received 27 July 1984 